\title{
Substrate regulation on co-metabolic degradation of $\beta$-cypermethrin by Bacillus licheniformis B-1
}

\author{
Jiayuan Zhao ${ }^{2}$, Dongying Jia ${ }^{1}$, Juan Du ${ }^{3}$, Yuanlong $\mathrm{Chi}^{1}$ and Kai Yao ${ }^{{ }^{*}}$
}

\begin{abstract}
Beta-cypermethrin ( $\beta-C Y$ ) residues are a serious threat to food safety and human health. However, the residues are not efficiently biodegraded because microorganisms preferentially use the nutrients found in food and the environment for growth. In this study, the mechanisms underlying nutrient regulation during co-metabolic degradation of $\beta-C Y$ by Bacillus licheniformis B-1 were investigated. The strain B-1 resting cells and the suspension containing $\mathrm{NaN}_{3}$ showed no significant differences in $\beta-C Y$ degradation. The co-metabolic degradation and strain B-1 growth could be separately inhibited by iodoacetic acid and sodium fluoride. Adenosine monophosphate (AMP), fructose 1-6 bisphosphate (F1-6BP), $\mathrm{Mg}^{2+}$, and $\mathrm{Mn}^{2+}$ could improve the degradation, whereas adenosine triphosphate (ATP), alanine (Ala), phenylalanine (Phe), and phosphoenolpyruvate (PEP) were found to exert the opposite effect, indicating that $\beta-C Y$ degradation was positively associated with pyruvate kinase activity. Furthermore, glycerol, urea, ammonium chloride and peptone improved $\beta$-CY degradation in corn flour. The results provided a promising approach for nutrient regulation of pyrethroids biodegradation in food and the environment.
\end{abstract}

Keywords: Beta-cypermethrin, Biodegradation, Co-metabolism, Substrate regulation, Bacillus licheniformis B-1

\section{Introduction}

Various studies have focused on the degradation of organic contaminants by microorganisms because of advantages, including potential eco-friendly safety and practical applicability (Deng et al. 2015; Hussain et al. 2016; Tang et al. 2018; Zhao et al. 2015, 2016, 2018). Organic contaminants are degraded by microorganisms through co-metabolism, the primary mechanism for degradation, and mineralization (Luo et al. 2014; Tran et al. 2013). Many organic contaminants could be degraded by microorganisms via co-metabolism, but the degradation of some compounds was no efficient (Luo et al. 2014; Tran et al. 2013). Generally, co-metabolism is the process in which the obligatory presence of a growth substrate or another utilizable compound is critically needed to maintain biomass and induce the corresponding enzyme and/

\footnotetext{
*Correspondence: yaokai555@126.com

${ }^{1}$ College of Light Industry, Textile \& Food Engineering, Sichuan University,

Chengdu 610065, Sichuan, People's Republic of China

Full list of author information is available at the end of the article
}

or cofactors for biodegradation (Tran et al. 2013). Therefore, the growth substrate is the key factor influencing co-metabolic degradation of organic contaminants by microorganisms.

Given that the use of organochlorine and organophosphorus pesticides has been totally or partly prohibited, pyrethroids have been widely used in agriculture and home formulations, accounting for about $25 \%$ of the worldwide pesticide market (Chen et al. 2013; Liu et al. 2014). Beta-cypermethrin ( $\beta-C Y)$ is an important pyrethroid that accounts for more than $50 \%$ of the total production of pyrethroids market in China (Xiao et al. 2015). $\beta$-CY residues are organic contaminants that accumulate in human and animal bodies through the food supply chain, thereby causing toxic effects on the reproductive, immune, and nervous systems and gene expression (Jin et al. 2011; Mckinlay et al. 2008; Schettgen et al. 2002). To eliminate or reduce the levels of $\beta-C Y$ residues in food and the environment, a large number of microorganisms such as Aspergillus niger YAT, Bacillus licheniformis B-1, Brevibacterium 
aureum, and Brevibacillus parabrevis BCP-09, which could degrade it through co-metabolism have been screened and isolated (Chen et al. 2013; Deng et al. 2015; Liu et al. 2014; Tang et al. 2018). However, the degradation efficiencies by these microorganisms in the food and environment were limited. Substrate regulation might be a viable method to co-metabolic degradation of organic contaminants by microorganisms (Liu et al. 2013; Luo et al. 2014; Nzila 2013). Loh and Wang (1997) reported that sodium glutamate could promote the degradation of 4-chlorophenol by Pseudomonas putida ATCC 49451, glucose supplementation significantly reduced its degradation. However, few studies have focused on investigating the regulatory mechanisms and pathway for co-metabolic degradation of organic contaminants by microbial nutrients.

The efficiencies of co-metabolic degradation of organic contaminants by microorganisms are influenced by energy dependence on the degradation (Luo et al. 2014), the glucose metabolism pathway for providing cofactors of degradation (Liu et al. 2013), and the characteristics of degradation-associated enzyme or degrading enzyme (Tran et al. 2013). Bacillus licheniformis B-1 was isolated from soil of tea garden and found to degrade $\beta$-CY through co-metabolism (Liu et al. 2014; Zhao et al. 2015, 2016, 2018). In this study, the characteristics of $\beta$-CY degradation by strain B-1 was investigated, the regulatory mechanisms and pathway for the co-metabolic degradation was analyzed, and substrate regulation approaches of $\beta-C Y$ degradation were verified in corn flour. The objectives of this study were to clarify the mechanisms underlying nutrition regulation for co-metabolic degradation of organic contaminants by microorganisms and to develop an efficient method for microbial co-metabolic degradation of organic contaminants present in food and the environment.

\section{Materials and methods Materials}

Beta-cypermethrin (99.7\%) and acetonitrile of chromatographic grade were obtained from the National Standard Substances Center (Beijing, China) and Meridian Medical Technologies (Beijing, China), respectively. Acetonitrile, ethyl acetate, ethyl alcohol, iodoacetic acid, sodium fluoride, glucose, glycerol, urea, $\mathrm{MgCl}_{2}, \mathrm{MnCl}_{2}$, alanine (Ala), phenylalanine (Phe), and $\left(\mathrm{NH}_{4}\right)_{2} \mathrm{SO}_{4}$ were of analytical grade and were procured from Kelong Chemical Co. (Chengdu, China). Nicotinamide adenine dinucleotide (NADH), adenosine monophosphate (AMP), fructose 1-6 bisphosphate (F1-6BP), adenosine triphosphate (ATP), and phosphoenolpyruvate (PEP) were purchased from Sigma.

\section{Microorganisms and media}

The strain Bacillus licheniformis B-1, which is capable of degrading $\beta$-CY via co-metabolism was isolated from the soil in a tea garden (Ya'an, China) (Liu et al. 2014; Zhao et al. 2015, 2016, 2018). Based on the analysis of morphological, physiological, biochemical characteristics and 16S rDNA sequence (GenBank Accession No.: HQ009796), strain B-1 was identified as Bacillus licheniformis (Liu et al. 2014), which has been stored in China Center for Type Culture Collection (NO. CCTCC M 2016258). Strain B-1 was stored in 15\% (v/v) glycerol solution at $-80^{\circ} \mathrm{C}$ before experiments.

Luria-Bertani (LB) medium containing $5.0 \mathrm{~g} / \mathrm{L}$ of yeast extract, $10.0 \mathrm{~g} / \mathrm{L}$ of peptone and $10.0 \mathrm{~g} / \mathrm{L}$ of $\mathrm{NaCl}$ was prepared. LB-CY medium consisted of LB medium and $\beta-\mathrm{CY}(100 \mathrm{mg} / \mathrm{L})$. The $\mathrm{pH}$ value of each medium was adjusted to $7.0-7.5$, and $0.2 \%(\mathrm{v} / \mathrm{v})$ of ethyl alcohol was added into the medium as a hydrotropic agent before sterilization at $121{ }^{\circ} \mathrm{C}$ for $20 \mathrm{~min}$ (Liu et al. 2014; Zhao et al. 2015).

\section{Inoculum preparation}

Strain B-1 was thawed and inoculated into a $100 \mathrm{~mL}$ Erlenmeyer flask, which contained $30 \mathrm{~mL}$ LB medium with $100 \mathrm{mg} / \mathrm{L} \beta$-CY. Then the flask was placed in a rotary shaker (Multitron-Pro, Infors, Switzerland) at $180 \mathrm{rpm}$ and $30{ }^{\circ} \mathrm{C}$, and the activated strain was obtained at $16 \mathrm{~h}$ cultivation. After being centrifuged (ST40R, Thermo, German) at $10,000 \mathrm{rpm}$ and $4{ }^{\circ} \mathrm{C}$ for $10 \mathrm{~min}$, the strain was collected and suspended in sterile $\mathrm{N}$-saline $(0.9 \%$ $\mathrm{NaCl})$ to achieve the cell density of about $1.0 \times 10^{8}$ cells/ $\mathrm{mL}$. Then, the bacterial suspension was used as inoculum (Liu et al. 2014; Zhao et al. 2015). The resting cells of strain B-1 were obtained after allowing the inoculum to stand for $48 \mathrm{~h}$ at $30^{\circ} \mathrm{C}$ (Sabullah et al. 2016).

\section{Determination of $\beta-\mathrm{CY}$ concentration}

The $\beta$-CY concentration was determined as previously described (Liu et al. 2014; Zhao et al. 2015). In detail, $5 \mathrm{~mL}$ of LB-CY medium and acetonitrile were transferred into a $100 \mathrm{~mL}$ flask and shaken for $30 \mathrm{~s}$ by a vortex mixer. Then, the flask was under ultrasonic $(40 \mathrm{kHz}$ and $300 \mathrm{~W}$ ) for $30 \mathrm{~min}$ (Liu et al. 2012). After the mixture was centrifuged at $8000 \mathrm{rpm}$ for $20 \mathrm{~min}$, the supernatant was collected and filtered through a $0.22 \mu \mathrm{m}$ membrane filter. Finally, the obtained filtrate was used to determine its $\beta$-CY content by high-performance liquid chromatograph (HPLC). The content of $\beta-C Y$ was determined by LC-20AT HPLC (Shimadzu, Kyoto, Japan) equipped with LC-20AT pump (Shimadzu), a CTO-20A column oven (Shimadzu), a Kromasil $\mathrm{C}_{18}$ column $(250 \mathrm{~mm} \times 4.60 \mathrm{~mm}$, 
$5.0 \mu \mathrm{m}$; Sweden) and an SPD-M20A detector. Degradation of $\beta-C Y$ was calculated according to the following equation:

$$
\text { Degradation }(\%)=\left(1-\mathrm{C} / \mathrm{C}_{0}\right) \times 100
$$

where $C$ and $C_{0}$ are the concentration of $\beta-C Y(\mathrm{mg} / \mathrm{L})$ in inoculated medium and the control, respectively.

\section{Energy dependence degradation assay}

The growth substances metabolism provided energy or co-factors for microorganisms to degrade organic contaminants via co-metabolism (Luo et al. 2014; Nzila 2013; Tran et al. 2013). To elucidate the energy dependence in the co-metabolic degradation of $\beta-C Y$, the degradation by resting cells of strain B-1, strain B-1 suspension, and strain B-1 suspension containing sodium azide $\left(\mathrm{NaN}_{3}\right)$ were investigated. For the energy dependence degradation assay, three samples, namely, the suspension of strain B-1, the suspension of strain B-1 with the inhibitors of $30 \mathrm{mmol} / \mathrm{L} \mathrm{NaN}_{3}$, and strain B-1 resting cells, were used to confirm whether the co-metabolic degradation of $\beta-C Y$ was energy-dependent (Hua et al. 2013; Sabullah et al. 2016). The initial $\beta-C Y$ concentration in all three samples was $30 \mathrm{mg} / \mathrm{L}$. Samples were incubated at $180 \mathrm{rpm}$ and $30^{\circ} \mathrm{C}$ for $12 \mathrm{~h}$ and subsequently analyzed as described above.

\section{Effect of glucose metabolism inhibitors on co-metabolic degradation of $\beta-C Y$ by strain B-1}

The energy or co-factors required by co-metabolic degradation of organic contaminants were from the microbial metabolism (Luo et al. 2014; Nzila 2013; Tran et al. 2013). Therefore, it is necessary to study the correlation between glucose metabolism and co-metabolic degradation of $\beta-\mathrm{CY}$ by strain B-1. Sodium fluoride, iodoacetic acid, trisodium phosphate and malonic acid were selected as the inhibitors of glucose metabolism (Shiota 1957; Wright 1937; Tang et al. 2011). Afterwards, a series of LB-CY media containing each of the inhibitors at $500 \mathrm{mg} / \mathrm{mL}$ were prepared. A $28.5 \mathrm{~mL}$ volume of LB-CY medium containing each inhibitor was mixed with $1.5 \mathrm{~mL}$ of inoculum in a $250 \mathrm{~mL}$ flask. The flask was shaken $(180 \mathrm{rpm})$ at $30{ }^{\circ} \mathrm{C}$ for $72 \mathrm{~h}$. The LB-CY medium without the inhibitor was used as control. Degradation of $\beta$-CY was calculated by Eq. (1), and the $\mathrm{OD}_{600}$ was determined as previously described (Liu et al. 2014; Zhao et al. 2015).

\section{Characteristics of co-metabolic degradation of $\beta-\mathrm{CY}$ by strain B-1}

The co-factors or inhibitors involved in co-metabolism such as metallic ions, ATP, NADH, or some intermediated metabolites play an important role for $\beta-C Y$ degradation, and the effects of these compounds on the degradation is needed to further investigate. Strain B-1 suspensions containing $30 \mathrm{mg} / \mathrm{L} \beta-\mathrm{CY}$ was used in the experiment. As shown in Table $1, \mathrm{MnCl}_{2}, \mathrm{MgCl}_{2}, \mathrm{ZnCl}_{2}$, $\mathrm{CaCl}_{2}, \mathrm{ATP}, \mathrm{NADH}, \mathrm{PEP}, \mathrm{AMP}, \mathrm{F} 1-6 \mathrm{BP}, \mathrm{AMP}$, Ala, and Phe were added to the mixture, and the samples were prepared. Some metallic ions were the key factors for the actions of inhibitors and activators during co-metabolic degradation of organic contaminants (Deng et al. 2015; Guo et al. 2009), and $\mathrm{MgCl}_{2}$ was used in the experiment for addition of inhibitors and activators. The sample was shaken $(180 \mathrm{rpm})$ at $30{ }^{\circ} \mathrm{C}$ for $72 \mathrm{~h}$. The residual $\beta-\mathrm{CY}$ concentration was measured by HPLC, and the Eq. (1) was used to compute the degradation of $\beta-\mathrm{CY}$.

\section{Nutrient regulation of co-metabolic degradation of $\beta-\mathrm{CY}$ by strain B-1 in corn flour}

Corn flours were purchased from Guo Jiaqiao market, Chengdu, Sichuan province, China. Corn flour (30 g) was mixed with $19.5 \mathrm{~mL}$ of purified water, and a series of mixtures with varying concentrations $(2.0,4.0,6.0$, 8.0 and $10.0 \mathrm{~g} / \mathrm{kg}$ ) of glucose, glycerol, urea, ammonium chloride and peptone were prepared, respectively. The resulting mixtures were sterilized at $121{ }^{\circ} \mathrm{C}$ for $20 \mathrm{~min}$. Then, the $\beta-C Y$ and strain $\mathrm{B}-1$ suspensions were added to the mixtures to achieve final concentrations of $50 \mathrm{mg}$ $\beta$-CY per kg corn flours and $1.0 \times 10^{8}$ cells per gram corn flours. Samples were inoculated at $180 \mathrm{rpm}$ and $30^{\circ} \mathrm{C}$ for $72 \mathrm{~h}$. $\beta$-CY concentrations were measured following the methods of Liu et al. (2014) and Zhao et al. (2015), and the degradation was calculated.

\section{Statistical analysis}

Each experiment was performed three times. Data were expressed as the means \pm standard deviations of three replicates. All statistical analyses were performed using SPSS v 17.0 (SPSS Inc., Chicago, IL, USA).

\section{Results}

\section{Energy dependence on the degradation of $\beta-C Y$ by strain}

B-1

Energy-containing molecules, such as ATP or guanosine triphosphate (GTP), could be produced in microbial suspension (Chauhan et al. 2003; Errécalde et al. 2004), but not in resting cells or strain B-1 suspension containing $\mathrm{NaN}_{3}$, in which energy production is exhausted or blocked by $\mathrm{NaN}_{3}$ via inhibition of oxidative phosphorylation (Chauhan et al. 2003; Errécalde et al. 2004). As shown in Table 2, the strain B-1 suspension, resting cells, and strain $\mathrm{B}-1$ suspension containing $\mathrm{NaN}_{3}$ showed no significant differences in $\beta-C Y$ degradation. It indicated that $\beta-C Y$ degradation by strain $\mathrm{B}-1$ did not require energy. 


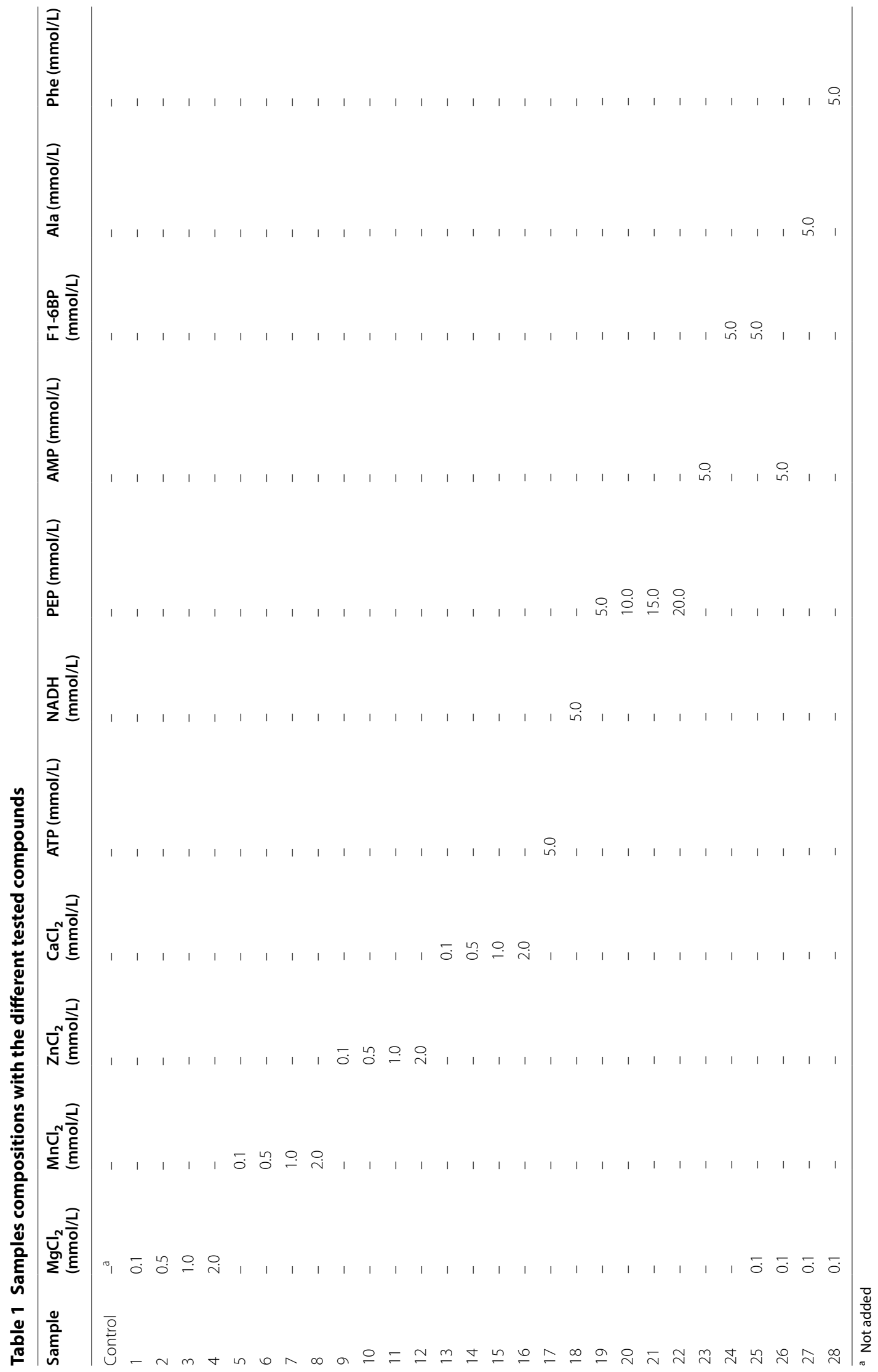


Table 2 Degradation of $\beta$-CY by resting cells and strain B-1 cells in suspension containing $\mathrm{NaN}_{3}$

\begin{tabular}{ll}
\hline Sample & $\boldsymbol{\beta}$-CY degradation (\%) \\
\hline Strain B-1 suspension & $23.42 \pm 0.36^{\mathrm{a}}$ \\
Resting cell & $23.35 \pm 0.28^{\mathrm{a}}$ \\
Strain B-1 suspension with $\mathrm{NaN}_{3}$ & $22.55 \pm 0.57^{\mathrm{a}}$
\end{tabular}

Data are presented as mean \pm standard deviation of three replicates; the standard deviations are within $5 \%$ of the mean. Different letters indicate significant differences $(p<0.05)$

\section{Effect of glucose metabolism inhibitors} on the co-metabolic degradation of $\beta-C Y$ by strain B-1 Some studies demonstrated that Embden-MeyerhofParnas (EMP) pathway, hexose monophosphate pathway (HMP) and tricarboxylic acid (TCA) cycle are the primary pathways involved in the glucose metabolism in Bacillus licheniformis (Calik and Ozdamar 1998; Voigt et al. 2004). Therefore, further studies are required to investigate the relationships among these pathways in glucose metabolism and $\beta$-CY degradation in strain B-1. Some compounds, such as iodoacetic acid and sodium fluoride, which inhibit the EMP pathway, and trisodium orthophosphate and malonic acid, which inhibit the HMP and TCA cycle, respectively, can additionally inhibit glucose metabolism (Shiota 1957; Wright 1937; Tang et al. 2011). The effects of glucose metabolism inhibitors on co-metabolic degradation of $\beta-\mathrm{CY}$ by strain B-1 in LB-CY medium are presented in Fig. 1. The degradation of $\beta-C Y$ and the $\mathrm{OD}_{600}$ of strain $\mathrm{B}-1$ in control samples were almost equal to those in the samples containing sodium phosphate and malonic acid, indicating that HMP and TCA cycle in strain B-1 were not correlated with the growth of cells and co-metabolic degradation of $\beta-C Y$. Nevertheless, the degradation of $\beta-C Y$ in the samples containing iodoacetic acid and sodium fluoride significantly deceased to $20.25 \%(p<0.05)$ and $0.15 \%$ $(p<0.01)$, respectively. The $\mathrm{OD}_{600}$ value of strain $\mathrm{B}-1$ in these two samples decreased by $38.61 \%$ and $32.05 \%$ relative to the control, respectively. The above results demonstrated that co-metabolic degradation of $\beta-C Y$ was correlated with EMP of strain B-1 in LB medium.

\section{Characteristics of the co-metabolic degradation of $\beta-C Y$ by strain B-1}

To investigate the characteristics of the organic contaminant degradation, the compounds involved in the HMP and TCA were used to regulate the degradation describing by Liu et al. (2013) and Lu et al. (2018), respectively. Some researches also indicated that the biodegradation of organic contaminants by

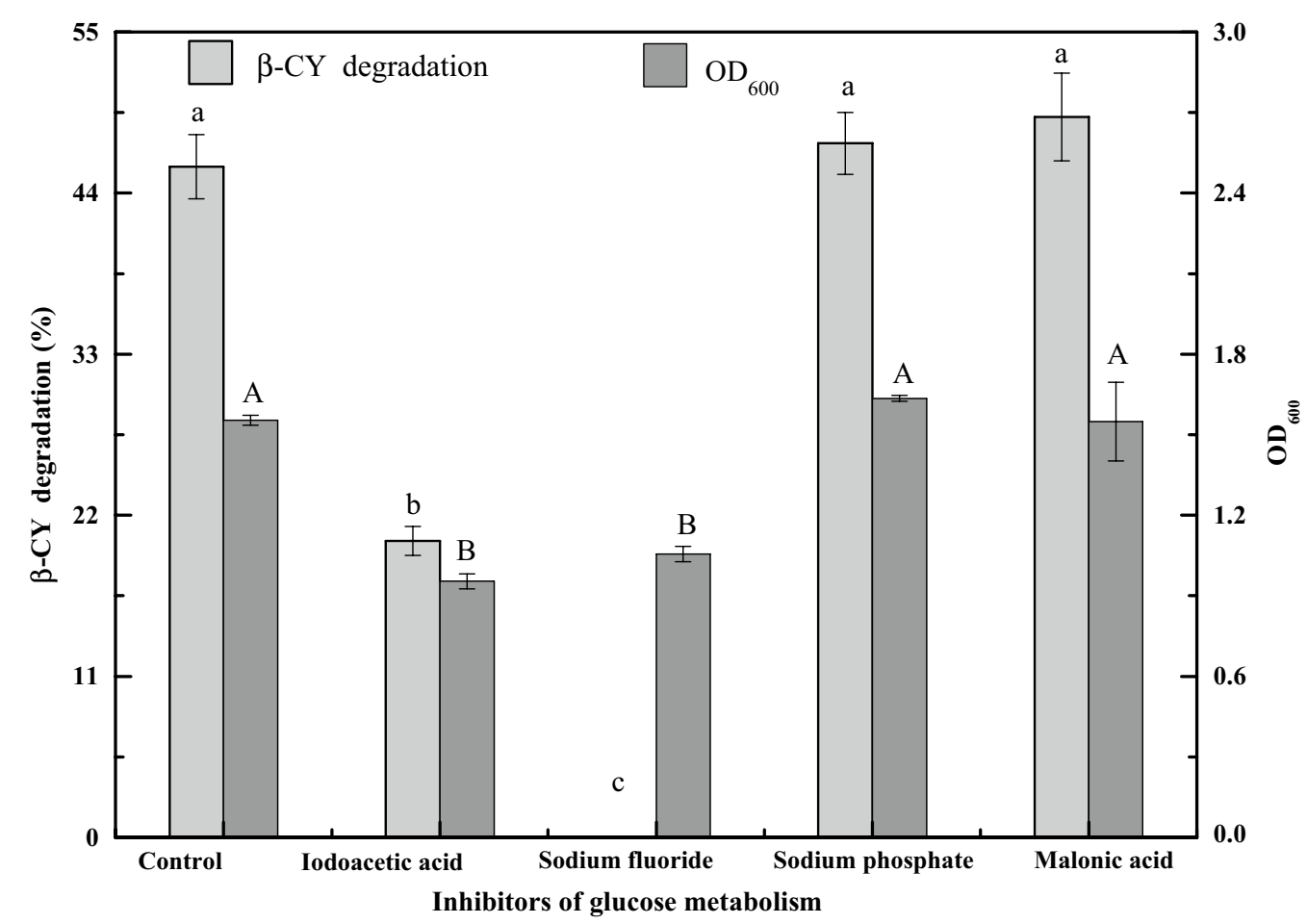

Fig. 1 Effects of glucose metabolism inhibitors on co-metabolic degradation of $\beta$-CY by strain B-1. Data are presented as mean \pm standard deviation of three replicates; the standard deviations are within $5 \%$ of the mean. Different letters indicate significant differences $(p<0.05)$ 
microorganisms or the growth of cells could be significantly affected by adding the compounds required by the glucose metabolism pathway (Gong et al. 2008; Hwang et al. 2011; Ostengo et al. 2005). Therefore, the relationship between the EMP in strain B-1 and the cometabolic degradation of $\beta-C Y$ was necessary to investigate, and the effects of specific compounds involved in the EMP on the co-metabolic degradation of $\beta$-CY were evaluated.

\section{Effects of divalent metal ions on the co-metabolic degradation of $\beta-C Y$}

The effects of $\mathrm{Mg}^{2+}, \mathrm{Mn}^{2+}, \mathrm{Zn}^{2+}$ and $\mathrm{Ca}^{2+}$ on the cometabolic degradation of $\beta-C Y$ by strain $\mathrm{B}-1$ suspension are shown in Table 3. Results indicated that $\mathrm{Mg}^{2+}$ and $\mathrm{Mn}^{2+}$ could improve the co-metabolic degradation of $\beta-C Y$ under optimal conditions. The degradation of $\beta-C Y$ was found to increase when the $\mathrm{Mg}^{2+}$ and $\mathrm{Mn}^{2+}$ concentrations were increased from 0.1 to $0.5 \mathrm{mmol} / \mathrm{L}$. At cation concentrations higher than $0.5 \mathrm{mmol} / \mathrm{L}, \beta-\mathrm{CY}$ degradation was found to decrease with increasing concentrations of $\mathrm{Mg}^{2+}$ and $\mathrm{Mn}^{2+}$. Further, the degradation of $\beta$-CY was almost constant in samples containing $\mathrm{Zn}^{2+}$ and $\mathrm{Ca}^{2+}$, indicating that these two metal ions did not significantly affect the co-metabolic degradation of $\beta-C Y$.

Table 3 Degradation of $\beta-C Y$ by strain B-1 cells in suspension after the addition of metallic ions

\begin{tabular}{lll}
\hline Metallic ions & $\begin{array}{l}\text { Concentrations } \\
\text { (mmol/L) }\end{array}$ & $\beta$-CY degradation (\%) \\
\hline Control & & $22.19 \pm 0.075^{\mathrm{a}}$ \\
$\mathrm{Mg}^{2+}$ & 0.1 & $26.14 \pm 1.47^{\mathrm{b}}$ \\
& 0.5 & $29.51 \pm 2.19^{\mathrm{b}}$ \\
& 1.0 & $22.58 \pm 1.72^{\mathrm{a}}$ \\
& 2.0 & $21.63 \pm 1.42^{\mathrm{a}}$ \\
$\mathrm{Mn}^{2+}$ & 0.1 & $27.03 \pm 0.92^{\mathrm{b}}$ \\
& 0.5 & $28.98 \pm 2.21^{\mathrm{b}}$ \\
& 1.0 & $25.46 \pm 1.43^{\mathrm{b}}$ \\
$\mathrm{Zn}^{2+}$ & 2.0 & $22.59 \pm 1.23^{\mathrm{a}}$ \\
& 0.1 & $22.70 \pm 0.23^{\mathrm{a}}$ \\
& 0.5 & $22.58 \pm 1.41^{\mathrm{a}}$ \\
& 1.0 & $21.09 \pm 0.92^{\mathrm{a}}$ \\
$\mathrm{Ca}^{2+}$ & 2.0 & $20.71 \pm 2.31^{\mathrm{a}}$ \\
& 0.1 & $21.92 \pm 2.12^{\mathrm{a}}$ \\
& 0.5 & $21.79 \pm 1.63^{\mathrm{a}}$ \\
& 1.0 & $21.09 \pm 1.12^{\mathrm{a}}$ \\
& 2.0 & $20.12 \pm 1.53^{\mathrm{a}}$
\end{tabular}

Data are presented as mean \pm standard deviation of three replicates; the standard deviations are within $5 \%$ of the mean. Different letters indicate significant differences $(p<0.05)$
Effects of ATP, NADH, and PEP on the co-metabolic degradation of $\beta-C Y$

The effects of ATP and NADH on co-metabolic degradation of $\beta-\mathrm{CY}$ are presented in Table 4. $\beta$-CY degradation in samples with ATP were observed to be lower compared to those in the control, while $\beta-C Y$ degradation in samples with NADH were almost constant. Therefore, ATP could inhibit the activity of the degradation-associated enzyme of strainB-1.

Co-metabolic degradation of $\beta-\mathrm{CY}$ after the addition of varying PEP concentrations are shown in Table 5 . The degradation of $\beta-C Y$ was found to decrease with increasing PEP concentrations, indicating that PEP could inhibit the co-metabolic degradation of $\beta$-CY.

Taken together, the above findings showed that the $\beta-C Y$ degradation-associated enzyme was associated with the EMP. In particular, PEP inhibit the co-metabolic degradation. Furthermore, $\beta-\mathrm{CY}$ degradation could be promoted by the addition of $\mathrm{Mg}^{2+}$ and $\mathrm{Mn}^{2+}$, but can be inhibited by ATP, thereby suggesting that pyruvate kinase is the $\beta$-CY degradation-associated enzyme.

\section{Verification of the degradation-associated enzyme in strain B-1}

To verified whether pyruvate kinase is the $\beta-C Y$ degradation-associated enzyme, the effects of activators and inhibitors of pyruvate kinase on the co-metabolic degradation of $\beta-C Y$ were investigated.

\section{Effects of pyruvate kinase activators on co-metabolic degradation of $\beta-C Y$}

Adenosine monophosphate (AMP), $\mathrm{Mg}^{2+}$ and F1-6BP were used as the pyruvate kinase activators (Tuominen and Bernlohr 1971). The effects of these activators on the co-metabolic degradation of $\beta-C Y$ by strain $B-1$ suspension are shown in Fig. 2. These activators were observed to improve $\beta-C Y$ degradation relative to the control. More specifically, $\beta-\mathrm{CY}$ degradation was found to be more efficient in samples containing AMP than

Table 4 Degradation of $\beta-C Y$ by strain B-1 after the addition of NADH and ATP

\begin{tabular}{|c|c|c|}
\hline Sample & $\begin{array}{l}\text { Concentration } \\
(\mathrm{mmol} / \mathrm{L})\end{array}$ & $\beta$-CY degradation (\%) \\
\hline Control & & $23.42 \pm 0.36^{\mathrm{a}}$ \\
\hline $\begin{array}{l}\text { Strain B-1 suspension with } \\
\text { NADH }\end{array}$ & 5.0 & $23.35 \pm 0.28^{a}$ \\
\hline $\begin{array}{l}\text { Strain B-1 suspension with } \\
\text { ATP }\end{array}$ & 5.0 & $19.85 \pm 0.57^{b}$ \\
\hline
\end{tabular}

Data are presented as mean \pm standard deviation of three replicates; the standard deviations are within $5 \%$ of the mean. Different letters indicate significant differences $(p<0.05)$ 
Table 5 Degradation of $\beta-C Y$ by strain B-1 after the addition of different concentrations of PEP

\begin{tabular}{ll}
\hline PEP concentrations (mmol/L) & $\boldsymbol{\beta}$-CY degradation (\%) \\
\hline Control & $22.68 \pm 1.28^{\mathrm{a}}$ \\
5.0 & $22.09 \pm 2.12^{\mathrm{a}}$ \\
10.0 & $17.01 \pm 1.06^{\mathrm{b}}$ \\
15.0 & $14.52 \pm 1.24^{\mathrm{c}}$ \\
20.0 & $12.31 \pm 1.10^{\mathrm{d}}$ \\
\hline
\end{tabular}

Data are presented as mean \pm standard deviation of three replicates; the standard deviations are within $5 \%$ of the mean. Different letters indicate significant differences $(p<0.05)$

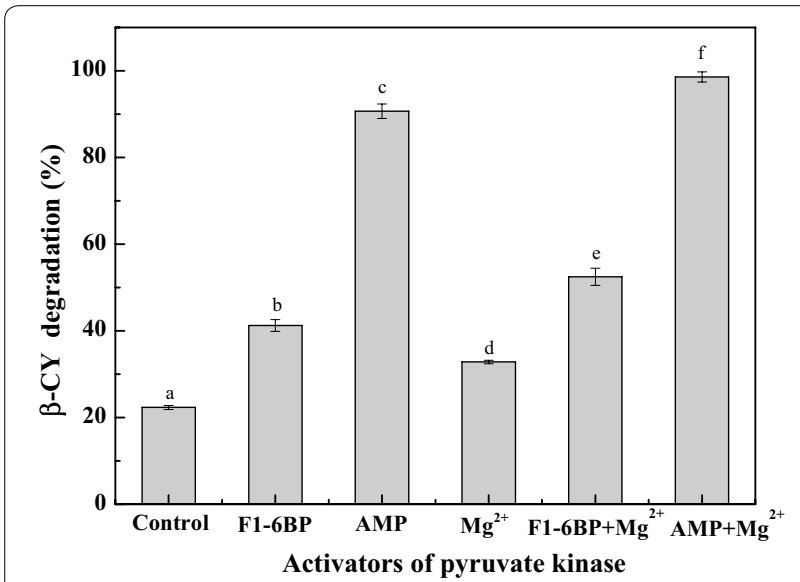

Fig. 2 Effects of pyruvate kinase activators on co-metabolic degradation of $\beta-C Y$ by strain B-1. Data are presented as mean \pm standard deviation of three replicates; the standard deviations are within $5 \%$ of the mean. Different letters indicate significant differences $(p<0.05)$

those in samples containing F1-6BP or $\mathrm{Mg}^{2+}$. Furthermore, samples containing AMP and $\mathrm{Mg}^{2+}$ showed fivefold higher peak degradation than those in the control.

\section{Effects of pyruvate kinase inhibitors on the co-metabolic degradation}

Alanine and Phe are generally used as inhibitors of pyruvate kinase (Tuominen and Bernlohr 1971; Zheng et al. 2014). The effects of Ala and Phe on the co-metabolic degradation of $\beta-C Y$ are shown in Fig. 3. Both of Ala and Phe could inhibit the degradation; however, samples with Phe showed higher degradation than that of samples containing Ala. The observed effects of activators and inhibitors of pyruvate kinase on the cometabolic degradation of $\beta-C Y$, evidently demonstrated that the activity of pyruvate kinase was positively associated with $\beta-C Y$ degradation by strain B-1.
Nutrient regulation of co-metabolic degradation of $\beta-\mathrm{CY}$ by strain B-1 in corn flour

The applications of corn in food were always restricted because of the presence of pesticide residues and its low protein content (Fields and Yoa 1990; Sharp et al. 1988). Bacillus licheniformis has been used to increase the protein content of corn flour (Fields and Yoa 1990). However, there is limited knowledge on $\beta-C Y$ degradation in corn flour. In this study, Bacillus licheniformis B-1 was used to degrade $\beta-C Y$ in corn flour, and the nutrient regulation during co-metabolic degradation of $\beta-C Y$ was verified by the addition of different nutrients which could regulate the activity of pyruvate kinase (Fig. 4).

\section{Effect of carbon sources on the co-metabolic degradation of $\beta-\mathrm{CY}$}

The effects of various concentrations of glucose and glycerol on the co-metabolic degradation of $\beta-C Y$ are shown in Fig. 5a. $\beta-\mathrm{CY}$ degradation decreased with higher concentrations of glucose. The degradation of $\beta-C Y$ increased with increasing concentrations of glycerol at concentrations lower than $6.0 \mathrm{~g} / \mathrm{kg}$. By contrast, at glycerol concentrations ranging from 6.0 to $10.0 \mathrm{~g} / \mathrm{kg}$, the degradation decreased from 72.96 to $42.30 \%$. These results suggested that the addition of optimal carbon sources in food could regulate and improve the $\beta-C Y$ degradation by microorganisms through co-metabolism.

\section{Effect of nitrogen sources on co-metabolic degradation of $\beta-\mathrm{CY}$}

The effects of urea, ammonium chloride and peptone on the co-metabolic degradation are shown in Fig. 5b. All three nitrogen sources were found to improve the co-metabolic degradation of $\beta-C Y$ by strain $\mathrm{B}-1$ was observed to increase with higher concentrations of urea and peptone. At a concentration of $10 \mathrm{~g} / \mathrm{kg}$, the $\beta-\mathrm{CY}$ degradation peaked at $79.44 \%$ and $85.99 \%$ for urea and peptone, respectively. In addition, $\beta$-CY degradation was slightly higher when the ammonium chloride concentrations were lower than $6.0 \mathrm{~g} / \mathrm{kg}$, whereas opposite results were obtained at ammonium chloride concentrations higher than $6.0 \mathrm{~g} / \mathrm{kg}$. These results indicated that urea, peptone and ammonium chloride could improve the cometabolic degradation of $\beta-C Y$ by strain B-1 in food.

\section{Discussion}

In order to efficiently eliminate the pesticides residues in the food and environment, lots of microorganisms have been screened and isolated, and further studies mainly focused on the pathway for pesticides degradation, the characteristics of degrading-enzymes, and the cloning of degradation genes (Liu et al. 2014; Zhao et al. 2015, 2016, 


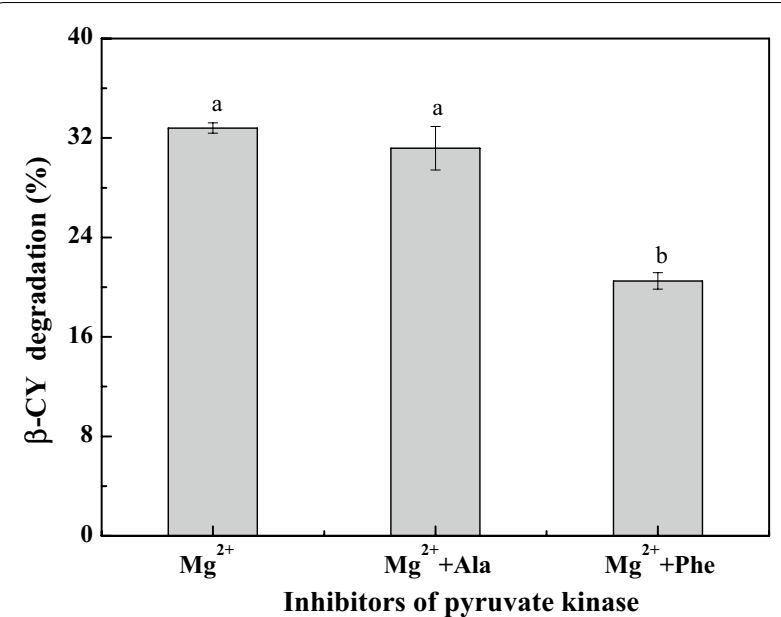

Fig. 3 Effect of pyruvate kinase inhibitors on co-metabolic degradation of $\beta-C Y$ by strain B-1. Data are presented as mean \pm standard deviation of three replicates; the standard deviations are within $5 \%$ of the mean. Different letters indicate significant differences $(p<0.05)$

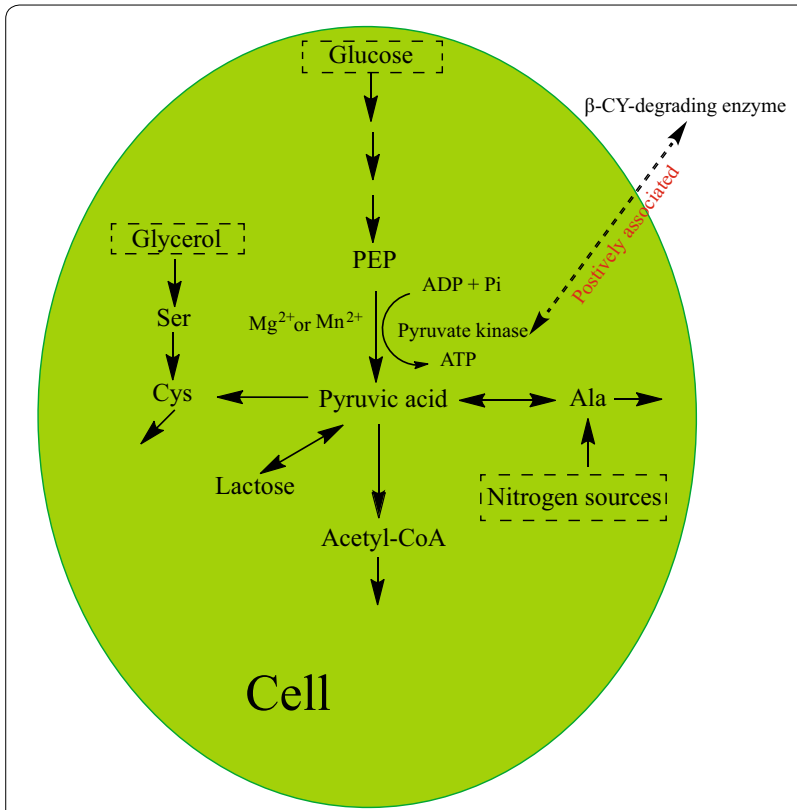

Fig. 4 Proposed pathway for nutrient regulation during co-metabolic degradation of $\beta-C Y$ by strain B-1. Co-metabolic degradation of $\beta-C Y$ by strain B-1 was positively associated with the activity of pyruvate kinase, which could be regulated by glucose, glycerol, and nitrogen sources

2018). Bacillus licheniformis B-1 could not use $\beta-C Y$ as sole source of carbon (Additional file 1: Fig. S1), indicating it degraded $\beta$-CY via co-metabolism. In this work, we firstly investigated the substrate regulation on cometabolic degradation of $\beta$-cypermethrin by strain B-1.
One of the interesting finding was that the degradation of $\beta-C Y$ by strain B-1 did not consume energy. Previous studies reported similar findings, in which the degradation enzymes produced by Aspergillus niger ZD11 and Sphingobacterium sp. JZ-2 were demonstrated to be capable of degrading $\beta-C Y$ without requiring energy containing molecules (Guo et al. 2009; Liang et al. 2005). Sabullah et al. (2016) also reported that glyphosate could be degraded by the resting cells of Klebsiella oxytoca, demonstrating that glyphosate degradation did not consume energy. However, some reports indicated that co-metabolism by microorganisms requires energy for breaking chemical bonds in the stable chemical structures of organic contaminants (Luo et al. 2014; Nzila 2013; Tran et al. 2013). This is because energy dependence of the degradation of organic contaminants was dependent on the types of organic contaminants and microorganisms (Hatzinger et al. 2017; Ji et al. 2017). The explanation for above findings is that $\beta$-CY degradation by microorganisms occurs via hydrolysis, in which the ester bond is rarely involved in energy metabolism.

Enzymes required for co-metabolic degradation of organic contaminants simultaneously participate in the degradation and the metabolism of carbon source (Luo et al. 2014; Nzila 2013). It has been reported that strain B-1 degrade $\beta$-CY via co-metabolism (Liu et al. 2014; Zhao et al. 2015, 2016, 2018). Therefore, it can be speculated an association between the carbon metabolism of strain B-1 and the co-metabolic degradation of $\beta-C Y$. The results indicated the degradation was correlated with EMP of strain B-1, because iodoacetic acid and sodium fluoride inhibit the activity of glyceraldehyde dehydrogenase phosphate and enolase the of EMP, respectively (Ostengo et al. 2005). Liu et al. (2013) reported that the co-metabolic degradation of imidacloprid was associated with hexose monophosphate pathway (HMP) in Stenotrophomonas maltophilia. Wang et al. (2019) indicated that the enzymes in TCA cycle of strain Rhodococcus sp. BAP-1 was related with degradation of fluoranthene by iTRAQ-based comparative proteomic analysis. Thereby, the compounds involved in EMP could affect the $\beta-C Y$ degradation.

The addition of certain divalent metal ions is known to increase the activity of some enzymes in the EMP (Calik and Ozdamar 1998; Voigt et al. 2004). The results indicated that higher concentrations of $\mathrm{Mg}^{2+}$ and $\mathrm{Mn}^{2+}$ impaired the biodegradation of $\beta-C Y$, which was attributed to the inhibiting cell growth (Tang et al. 2011). While, low concentrations of $\mathrm{Mg}^{2+}$ and $\mathrm{Mn}^{2+}$ improved the $\beta$-CY degradation. Yi et al. (2012) reported that the activity of the pyrethroid-degrading enzyme in Ochrobactrum anthropi YZ-1 was increase by $17.60 \%$ when the $\mathrm{Mg}^{2+}$ and $\mathrm{Mn}^{2+}$ concentrations were set to $1.0 \mathrm{mmol} / \mathrm{L}$. 

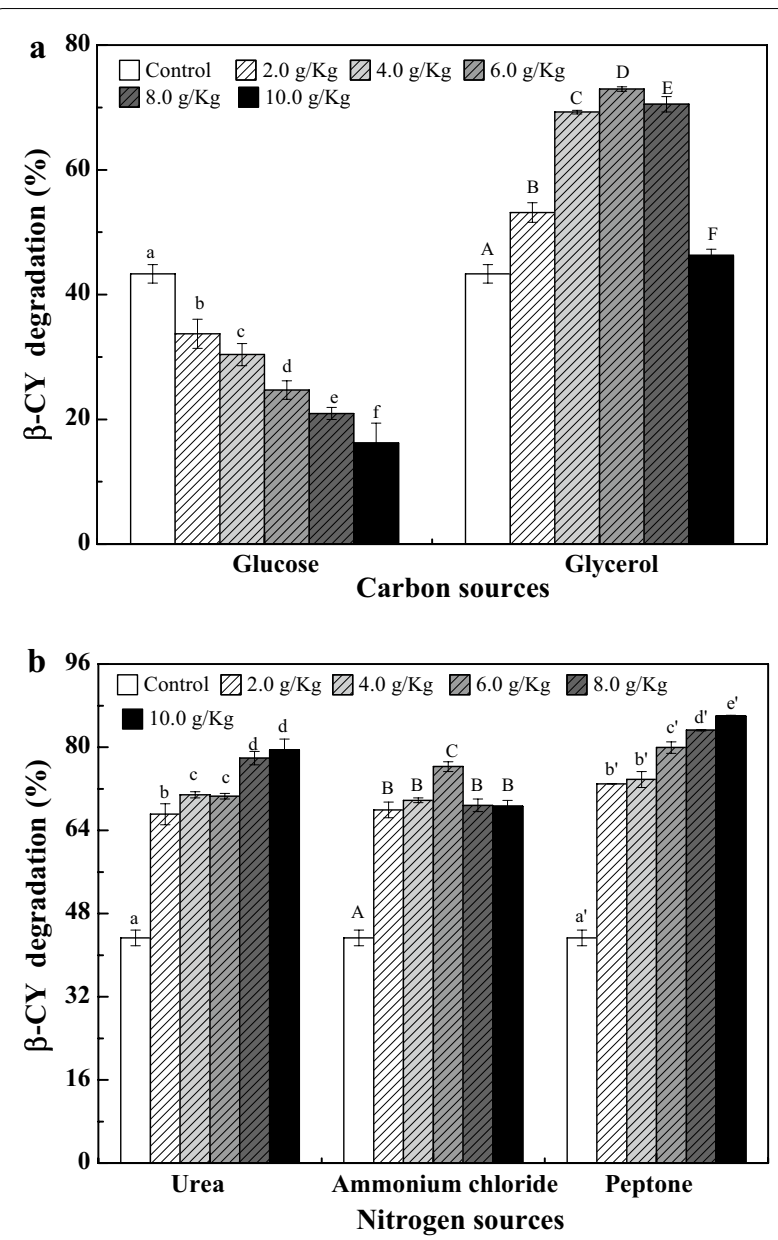

Fig. 5 Effects of the addition of carbon (a) and nitrogen sources (b) on co-metabolic degradation of $\beta-C Y$ in corn flour by strain B- 1 . Data are presented as mean \pm standard deviation of three replicates; the standard deviations are within $5 \%$ of the mean. Different letters indicate significant differences $(p<0.05)$

However, it is difficult to judge whether $\mathrm{Mg}^{2+}$ and $\mathrm{Mn}^{2+}$ promoted the activity of the degradation enzyme or the degradation-associated enzymes in strain B-1.

ATP and NADH are not only metabolites of the EMP, but additionally provide energy or serve as cofactors for co-metabolic enzymes (Calik and Ozdamar 1998; Voigt et al. 2004). Lu et al. (2018) reported that adding ATP and NADH could enhance the transformation of organic component. Our results indicated that ATP inhibited the $\beta$-CY degradation, while NADH did not significantly affect it. However, previous studies reported contradictory results, in which ATP or NADH were found to improve the degradation of organic contaminants by microorganisms (Luo et al. 2014; Nzila 2013; Tran et al. 2013) and was attributed to differences in the chemical structures of organic contaminants containing pyrethroid
(Luo et al. 2014). Moderate amounts of ATP are not toxic to microorganisms. Although enzymatic inhibition by ATP was unexpected, a possible explanation was that the co-metabolic degrading enzyme of $\beta$ - $\mathrm{CY}$ or the degradation-associated enzyme could utilize growth substances to produce ATP, which in turn inhibited enzymatic activity through feedback inhibition.

Above results indicated that the enzyme responsible for $\beta$-CY degradation in strain B-1 did not produce and consume ATP during co-metabolic degradation of $\beta-\mathrm{CY}$. Therefore, ATP could inhibit the activity of the degradation-associated enzyme of strainB-1. Moreover, AMP was speculated to promote $\beta-C Y$ degradation by strain B-1, which was validated in Fig. 2.

As an important metabolite of the EMP, phosphoenolpyruvate (PEP) could be metabolized by pyruvate kinase to produce ATP in the presence of $\mathrm{Mg}^{2+}$ and $\mathrm{Mn}^{2+}$ (Calik and Ozdamar 1998; Voigt et al. 2004). PEP showed negative effects on $\beta$-CY degradation. There are two possible explanations for the above results. First, PEP could act as the substrate of the $\beta$-CY degradation-associated enzyme, and the product (ATP) would inhibit the activity of the enzyme. Second, PEP could be the product of the $\beta$-CY degradation-associated enzyme, and can inhibit $\beta-C Y$ degradation via feedback inhibition.

The allosteric effect of AMP on the pyruvate kinase of Bacillus licheniformis is stronger than that of F1-6BP (Tanaka et al. 1995). Pyruvate kinase activators have different mechanisms of action (Tanaka et al. 1995; Tuominen and Bernlohr 1971; Zheng et al. 2014), resulting $\beta-C Y$ degradation in the samples containing F1-6BP, $\mathrm{Mg}^{2+}, \mathrm{F} 1-6 \mathrm{BP}$ and $\mathrm{Mg}^{2+}$ were $41.24 \%, 32.81 \%$ and $52.47 \%$, respectively. Meanwhile, the inhibiting effects of Ala and Phe were also different, which is attributed to the allosteric effect of Phe than that of Ala (Muñoz and Ponce 2003). Ala binds to the deep pocket of pyruvate kinase located between the $\mathrm{A}$ and $\mathrm{C}$ domains, which is distant from the active site; the binging of Phe to pyruvate kinase leads to higher flexibility of the protein structure (Muñoz and Ponce 2003; Williams et al. 2006; Zheng et al. 2014).

Favaloro et al. (2000) reported that glutathione synthase was degradation-associated enzyme for the cometabolic degradation of atrazine by Ochrobactrum anthropic, and its regulatory factors could affect atrazine degradation. For microorganisms via co-metabolism, the only way to regulate the efficiency of microbial degradation of organic pollutants present in foods was to investigate the characteristics of degrading enzyme or degradation-associated enzyme (Liu et al. 2013).

Taken together, previous findings and the current results indicated that $\beta$-CY degradation could be regulated by controlling the activity of pyruvate kinase, which was strongly influenced by the presence of 
specific nutrients. Based on previous finding on the metabolic pathways in Bacillus licheniformis (Voigt et al. 2004), the regulatory pathway for $\beta$-CY degradation is summarized in Fig. 4. $\beta$-CY degradation was positively associated with the activity of pyruvate kinase, the key enzyme involved in the EMP (Schettgen et al. 2002). It is well known that carbon and nitrogen sources that are associated with the EMP pathway can influence pyruvate kinase activity (Muñoz and Ponce 2003; Tuominen and Bernlohr 1971; Voigt et al. 2004; Zheng et al. 2014). Thus, the co-metabolic degradation of $\beta$-CY could be improved by the addition of nutrients that increase the activity of pyruvate kinase. As shown in Fig. 4, glucose, glycerol and nitrogen sources could effectively regulate the activity of pyruvate kinase.

In corn flour, the concentrations of glucose were negatively associated with $\beta$-CY degradation, because the glucose could be converted into PEP through the EMP (Voigt et al. 2004) and high concentrations of PEP would inhibit $\beta$-CY degradation (Table 5). Meanwhile, high concentrations of glycerol also could reduce the degradation of $\beta-C Y$; while, at low concentrations, it could improve the degradation. It is likely that Bacillus licheniformis preferentially utilizes glycerol than starch (Voigt et al. 2004) and the activity of pyruvate kinase can be enhanced at low glycerol concentrations. However, at higher glycerol concentrations, high concentrations of cysteine produced during glycerol metabolism inhibit enzymatic activity of pyruvate kinase. Similar results were reported by Liu et al. (2013) in sample containing sucrose, glucose, or fructose, which were observed to have significantly higher imidacloprid degradation. The results indicated that the degradation of $\beta-C Y$ was increased as the concentrations of urea and peptone increased. These findings are attributed to the relatively low nitrogen content in corn flour, and the addition of urea and peptone provided nitrogen sources for the growth of strain B-1 (Calik and Ozdamar 1998; Fields and Yoa 1990; Voigt et al. 2004). Although ammonium chloride also could improve the degradation, when the concentrations higher than $0.6 \mathrm{~g} / \mathrm{kg}$ the degradation decreased. It is likely that ammonium chloride could improve the growth of strain B-1 at lower concentrations; however, at higher ammonium chloride concentrations, large amounts of hydroxylamine can be produced by microbial nitrification and converted to phosphoramide via a hydroxylamine reaction, which in turn inhibits the growth of strain B-1 (Nowak and Suelter 1981). These results demonstrated the degradation of $\beta$-CY could be improved by regulating the activity of pyruvate kinase.

\section{Additional file}

Additional file 1: Fig. S1. Concentrations of $\beta-C Y$ in MS-CY medium for $72 \mathrm{~h}$ by strain B-1.

\section{Abbreviations}

$\beta$-CY: $\beta$-cypermethrin; NADH: nicotinamide adenine dinucleotide; ATP: adenosine triphosphate; GTP: guanosine triphosphate; AMP: adenosine monophosphate; PEP: phosphoenolpyruvate; F1-6BP: fructose 1,6 bisphosphate; Ala: alanine; Phe: phenylalanine; HPLC: high-performance liquid chromatograph; EMP: Embden-Meyerhof-Parnas; HMP: hexose monophosphate pathway; TCA cycle: tricarboxylic acid cycle.

\section{Acknowledgements}

Not applicable.

\section{Authors' contributions}

$J Z$ and KY conceived and designed this experiment. JZ and DJ performed the experiment and contributed to the data analysis and the writing of the manuscript. YC and JD performed the research and provided technical support. All authors read and approved the final manuscript.

\section{Funding}

This work was financially supported by the National Natural Science Foundation of China (Grant Numbers: 31371775 and 31801644), and the Specialized Fund for the Experimental Technology and Management Project of Sichuan Normal University of China (SYJS2018001).

\section{Availability of data and materials}

The dataset supporting the conclusions of this article is included within the article. All data are fully available without restriction.

\section{Ethics approval and consent to participate}

This article does not contain any studies with human participants or animals performed by any of the authors.

\section{Consent for publication}

Not applicable.

\section{Competing interests}

The authors declare that they have no competing interests.

\section{Author details}

${ }^{1}$ College of Light Industry, Textile \& Food Engineering, Sichuan University, Chengdu 610065, Sichuan, People's Republic of China. ${ }^{2}$ College of Life Science, Sichuan Normal University, Chengdu 610101, Sichuan, People's Republic of China. ${ }^{3}$ The Faculty Geography Resource Science, Sichuan Normal University, Chengdu 610101, Sichuan, People's Republic of China.

Received: 31 May 2019 Accepted: 6 June 2019

Published online: 12 June 2019

\section{References}

Calik P, Ozdamar TCG (1998) Oxygen transfer effects in serine alkaline protease fermentation by Bacillus licheniformis: use of citric acid as the carbon source. Enzyme Microb Technol 23(7-8):451-461

Chauhan VS, Singh B, Singh S, Bisen PS (2003) Regulation of potassium uptake in the sodium-resistant $(\mathrm{NaCl}(\mathrm{r}))$ and thalium-resistant $(\mathrm{TICl}(\mathrm{r}))$ mutant strain of diazotrophic cyanobacterium Anabaena variabilis. Curr Microbiol 46(1):0059-0064

Chen S, Dong YH, Chang C, Deng Y, Zhang XF, Zhong G, Song H, Hu M, Zhang LH (2013) Characterization of a novel cyfluthrin-degrading bacterial strain Brevibacterium aureum and its biochemical degradation pathway. Bioresour Technol 132(3):16-23

Deng W, Lin D, Yao K, Yuan H, Wang Z, Li J, Zou L, Han X, Zhou K, He L (2015) Characterization of a novel $\beta$-cypermethrin-degrading Aspergillus niger 
YAT strain and the biochemical degradation pathway of $\beta$-cypermethrin. Appl Microbiol Biotechnol 99(19):1-12

Errécalde O, Gommy C, Maury G, Pinel R (2004) Biotransformation of tributyltin chloride in the presence of resting-cell suspensions of pure strains of microorganisms. Appl Organomet Chem 9(7):525-529

Favaloro B, Tamburro A, Trofino MA, Bologna L, Rotilio D, Heipieper HJ (2000) Modulation of the glutathione S-transferase in Ochrobactrum anthropi: function of xenobiotic substrates and other forms of stress. Biochem J 346(Pt 2(2)):553

Fields ML, Yoa FG (1990) Nutritional improvement of corn meal by fermentation by Bacillus licheniformis and Enterobacter cloacae. J Food Prot 53(3):245-248

Gong F, Zhang T, Chi ZM, Sheng J, Li J, Wang XH (2008) Purification and characterization of extracellular inulinase from a marine yeast Pichia guilliermondii and inulin hydrolysis by the purified inulinase. Biotechnol Bioprocess Eng 13(5):533-539

Guo P, Wang B, Hang BJ, Li L, Ali SW, He J, Li S (2009) Pyrethroid-degrading Sphingobium sp. JZ-2 and the purification and characterization of a novel pyrethroid hydrolase. Int Biodeterior Biodegrad 63(8):1107-1112

Hatzinger PB, Banerjee R, Rezes R, Streger SH, Mcclay K, Schaefer CE (2017) Potential for cometabolic biodegradation of 1,4-dioxane in aquifers with methane or ethane as primary substrates. Biodegradation 28(1):1-16

Hua F, Wang H, Li Y, Zhao YC (2013) Trans-membrane transport of $n$-octadecane by Pseudomonas sp. DG17. J Microbiol 51(6):791-799

Hussain S, Hartley CJ, Shettigar M, Pandey G (2016) Bacterial biodegradation of neonicotinoid pesticides in soil and water systems. FEMS Microbiol Lett 363(23):1-13

Hwang CF, Jiang YS, Sheu SC, Hsieh PC, Guo JH (2011) Purification and characterization of a novel glucose oxidase-like melanoidin decolorizing enzyme from Geotrichum sp. No. 56. Afr J Microbiol Res 5(20):3256-3266

Ji X, Xu J, Ning S, Li N, Tan L, Shi S (2017) Cometabolic degradation of dibenzofuran and dibenzothiophene by a naphthalene-degrading Comamonas sp. JB. Curr Microbiol 74(12):1411-1416

Jin Y, Zheng S, Fu Z (2011) Embryonic exposure to cypermethrin induces apoptosis and immunotoxicity in zebrafish (Danio rerio). Fish Shellfish Immunol 30:1049-1054

Liang WQ, Wang ZY, Li H, Wu PC, Hu JM, Luo N, Cao LX, Liu YH (2005) Purification and characterization of a novel pyrethroid hydrolase from Aspergillus niger ZD11. J Agric Food Chem 53(19):7415-7420

Liu S, Yao K, Jia D, Zhao N, Lai W, Yuan H (2012) A pretreatment method for HPLC analysis of cypermethrin in microbial degradation systems. J Chromatogr Sci 50(6):469

Liu Z, Dai Y, Huan Y, Liu Z, Sun L, Zhou Q, Zhang W, Sang Q, Wei H, Yuan S (2013) Different utilizable substrates have different effects on cometabolic fate of imidacloprid in Stenotrophomonas maltophilia. Appl Microbiol Biotechnol 97(14):6537-6547

Liu F, Chi Y, Wu S, Jia D, Yao K (2014) Simultaneous degradation of cypermethrin and its metabolite, 3-phenoxybenzoic acid, by the cooperation of Bacillus licheniformis B-1 and Sphingomonas sp. SC-1. J Agric Food Chem 62(33):8256-8262

Loh KC, Wang SJ (1997) Enhancement of biodegradation of phenol and a nongrowth substrate 4-chlorophenol by medium augmentation with conventional carbon sources. Biodegradation 8(5):329-338

Lu Q, Zhao Y, Gao XT, Wu JQ, Zhou HX, Tang PF, Wei QB, Wei ZM (2018) Effect of tricarboxylic acid cycle regulator on carbon retention and organic component transformation during food waste composting. Bioresour Technol 256:128-136

Luo W, Zhu X, Chen W, Duan Z, Wang L, Zhou Y (2014) Mechanisms and strategies of microbial cometabolism in the degradation of organic compounds chlorinated ethylenes as the model. Water Sci Technol 69(10):1971-1983

Mckinlay R, Plant JA, Bell JNB, Voulvoulis N (2008) Endocrine disrupting pesticides: implications for risk assessment. Environ Int 34(2):168-183

Muñoz ME, Ponce E (2003) Pyruvate kinase: current status of regulatory and functional properties. Comp Biochem Physiol Part B Biochem Mol Biol 135(2):197

NowakT, Suelter C (1981) Pyruvate kinase: activation by and catalytic role of the monovalent and divalent cations. Mol Cell Biochem 35(2):65-75

Nzila A (2013) Update on the cometabolism of organic pollutants by bacteria. Environ Pollut 178:474-482

Ostengo MDA, Wiese B, Nader-Macias ME (2005) Inhibitory effect of sodium fluoride and chlorhexidine on the growth of oral lactobacilli. Can J Microbiol 51(2):133-140
Sabullah MK, Rahman MF, Ahmad SA, Sulaiman MR, Shukor MS, Shamaan NA, Shukor MYA (2016) Isolation and characterization of a molybdenumreducing and glyphosate-degrading Klebsiella oxytoca strain saw-5 in soils from sarawak. J Agric Sci 38(1):1-13

Schettgen T, Heudorf U, Drexler H, Angerer J (2002) Pyrethroid exposure of the general population—is this due to diet. Toxicol Lett 134:141-145

Sharp GJ, Brayan JG, Dilli S, Haddad PR, Desmarchelier JM (1988) Extraction, clean-up and chromatographic determination of organophosphate, pyrethroid and carbamate insecticides in grain and grain products. A review. Analyst 113(10):1493

Shiota T (1957) Effect of fluoride, iodoacetic acid, penicillin, sodium lauroyl sarcosinate and dehydroacetic acid on certain oral bacteria of white rats fed a smooth surface caries diet: preliminary report. J Am Dent Assoc 55(2):205-210

Tanaka K, Sakai H, Ohta T, Matsuzawa H (1995) Molecular cloning of the genes for pyruvate kinase of two bacilli, Bacillus psychrophilus and Bacillus licheniformis, and comparison of the properties of the enzymes produced in Escherichia coli. Biosci Biotechnol Biochem 59(8):1536

Tang Q, Zhou X, Lu Z, Wang Y, Bie X (2011) Effects of fengycin from Bacillus subtilis fmbJ on respiratory chain and nutrients utilization of Rhizopus stolonifer. Food Sci China 32(11):248-254

Tang J, Liu B, Chen TT, Yao K, Zeng L, Zeng CY, Zhang Q (2018) Screening of a beta-cypermethrin-degrading bacterial strain Brevibacillus parabrevis BCP-09 and its biochemical degradation pathway. Biodegradation 29(6):525-541. https://doi.org/10.1007/s10532-018-9850-0

Tran NH, Urase T, Ngo HH, Hu J, Ong SL (2013) Insight into metabolic and cometabolic activities of autotrophic and heterotrophic microorganisms in the biodegradation of emerging trace organic contaminants. Bioresour Technol 146:721-731

Tuominen FW, Bernlohr RW (1971) Pyruvate kinase of the spore-forming bacterium, Bacillus licheniformis. I. Purification, stability, regulation of synthesis, and evidence for multiple molecular states. J Biol Chem 246(6):1733

Voigt B, Schweder T, Becher D, Ehrenreich A, Gottschalk G, Feesche J, Maurer KH, Hecker M (2004) A proteomic view of cell physiology of Bacillus licheniformis. Proteomics 4(5):1465-1490

Wang HQ, Yang Y, Xu J, Kong DK, Li Y (2019) iTRAQ-based comparative proteomic analysis of differentially expressed proteins in Rhodococcus sp. BAP-1 induced by fluoranthene. Ecotoxicol Environ Saf 169:282-291

Williams R, HolyoakT, Mcdonald G, Chunshan Gui A, Fenton AW (2006) Differentiating a ligand's chemical requirements for allosteric interactions from those for protein binding. Phenylalanine inhibition of pyruvate kinase. Biochemistry 45(17):5421-5429

Wright HD (1937) The effect of iodoacetic acid, fluoride, phloridzin and phenol on bacterial fermentation: a further consideration of the fermentation of disaccharides. J Pathol Bacteriol 45(1):117-129

Xiao Y, Chen S, Gao Y, Hu W, Hu M, Zhong G (2015) Isolation of a novel betacypermethrin degrading strain Bacillus subtilis BSF01 and its biodegradation pathway. Appl Microbiol Biotechnol 99(6):2849-2859

Yi Z, Li K, Song J, Shi Y, Yan Y (2012) Molecular cloning, purification and biochemical characterization of a novel pyrethroid-hydrolyzing carboxylesterase gene from Ochrobactrum anthropi YZ-1. J Hazard Mater 221-222(4):206-212

Zhao J, Chi Y, Liu F, Jia D, Yao K (2015) Effects of two surfactants and betacyclodextrin on beta-cypermethrin degradation by Bacillus licheniformis B-1. J Agric Food Chem 63(50):10729

Zhao J, Chi Y, Xu Y, Jia D, Yao K (2016) Co-metabolic degradation of $\beta$-cypermethrin and 3-phenoxybenzoic acid by co-culture of Bacillus licheniformis B-1 and Aspergillus oryzae M-4. PLoS ONE 11(11):e0166796

Zhao J, Li X, Chi Y, Jia D, Yao K (2018) Characterization of co-culturing microorganisms for simultaneous degradation of beta-cypermethrin and 3-phenoxybenzoic acid. Fresenius Environ Bull 27(6):4249-4257

Zheng L, XU T, Bai Z, He B (2014) $\mathrm{Mn}^{2+} / \mathrm{Mg}^{2+}$-dependent pyruvate kinase from a D-lactic acid-producing bacterium Sporolactobacillus inulinus: characterization of a novel $\mathrm{Mn}^{2+}$-mediated allosterically regulated enzyme. Appl Microbiol Biotechnol 98(4):1583-1593

\section{Publisher's Note}

Springer Nature remains neutral with regard to jurisdictional claims in published maps and institutional affiliations. 